\title{
The heliospheric magnetic field and the solar wind during the solar cycle
}

\author{
Lennard A. Fisk and Liang Zhao \\ Dept. of Atmospheric, Oceanic and Space Sciences, University of Michigan, \\ 2455 Hayward St., Ann Arbor, Michigan 48109, USA \\ email: lafisk@umich.edu
}

\begin{abstract}
The heliospheric magnetic field and the solar wind are behaving differently in the current solar minimum, compared to the previous minimum. The radial component of the heliospheric magnetic field, and thus the average value of the component of the solar magnetic field that opens into the heliosphere, the so-called open magnetic flux of the Sun, is lower than it was in the previous solar minimum; in fact, lower than in any previous solar minimum for which there are good spacecraft observations. The mass flux, the ram pressure, and the coronal electron temperature as measured by solar wind charge states are also lower in the current minimum compared to the previous one. This situation provides an opportunity to test some of the concepts for the behavior of the heliospheric magnetic field and the solar wind that have been developed; to improve these theories, and to construct a theory for the solar wind that accounts for the observed behavior throughout the solar cycle, including the current unusual solar minimum.
\end{abstract}

Keywords. Solar magnetic field, heliospheric magnetic field, coronal processes, solar wind, solar cycle

\section{Introduction}

Over the last decade we have developed theories for the behavior of the heliospheric magnetic field (Fisk 1996; Zurbuchen et al. 1997; Fisk et al. 1999a,b; Fisk 1999; Fisk \& Schwadron 2001; Fisk 2005; Fisk \& Zurbuchen 2006), and for the acceleration of the solar wind (Fisk et al. 1998, 1999c; Fisk 2003). During the current solar minimum, the heliospheric magnetic field and the solar wind are behaving in ways that are different from any previous minimum for which we have good spacecraft observations. The current solar minimum thus provides a unique opportunity to test these theories, modify them as necessary, and construct theories that can account for the behavior of the heliospheric magnetic field and the solar wind throughout the solar cycle, including the current unusual minimum.

We begin by reviewing the observations of the heliospheric magnetic field and the solar wind, from the previous solar minimum through the present one, to illustrate the differences between the two successive minima. We use observations from Ulysses, which span the entire time period. We then consider whether our theories for the behavior of the heliospheric magnetic field are consistent with these observations, and suggest an addition to these theories that improves the consistency. We next develop a theory for the solar wind, based on our previous theories for the solar wind, which can account for the behavior of the solar wind mass flux, coronal electron temperatures and thus the ionic charge-states of the solar wind, and for the solar wind flow speed, and which is consistent with observations throughout the solar cycle. 


\section{Observations}

Summarized in Figure 1 are some of the main observations we will deal with:

Radial magnetic field strength. In the top panel is the radial component of the heliospheric magnetic field, normalized by heliocentric radial distance squared. We will refer to this as the normalized radial component of the heliospheric magnetic field. The measurements from Ulysses are from many different latitudes; however, it has been shown from Ulysses that the latitude variations in the heliospheric radial magnetic field are weak, as is to be expected (Smith \& Balogh 1995; Balogh \& Smith 2001). The magnetic pressure in the outer solar corona, where the magnetic field, dragged outward with the solar wind, is radial, must be constant since there are no latitudinal balancing forces; i.e., the radial magnetic field should be uniform. The radial component of the heliospheric magnetic field is thus a measure of the average value of the component of the solar magnetic field that opens into the heliosphere, the so-called open magnetic flux of the Sun.

Note that the normalized radial component varies over the solar cycle, increasing by a factor of $\sim 2$ near solar maximum, and it attains its minimum value in solar minimum. Note also that the minimum value of the normalized radial component is lower in the current solar minimum than it was in the previous one.

Solar wind mass flux. In the second panel of Figure 1 is the mass flux of the solar wind. Note that the mass flux roughly tracks the normalized radial component of the heliospheric magnetic field, and it too is lower in the current minimum than in the previous one.

Solar wind ram pressure. In the third panel of Figure 1 is shown the ram pressure of the solar wind. Note that it also roughly tracks the solar wind mass flux in the two successive minima. This indicates that although the mass flux is lower in the current minima, the range of solar wind flow speeds is not different between the two minima.

Solar wind charge state. In the bottom panel of Figure 1 is shown the ratio of $\mathrm{O}^{7+}$ to $\mathrm{O}^{6+}$ in the solar wind. Note that in the current solar minimum the ratio is lower than in the previous minimum. The charge states of the solar wind are frozen-in in the solar

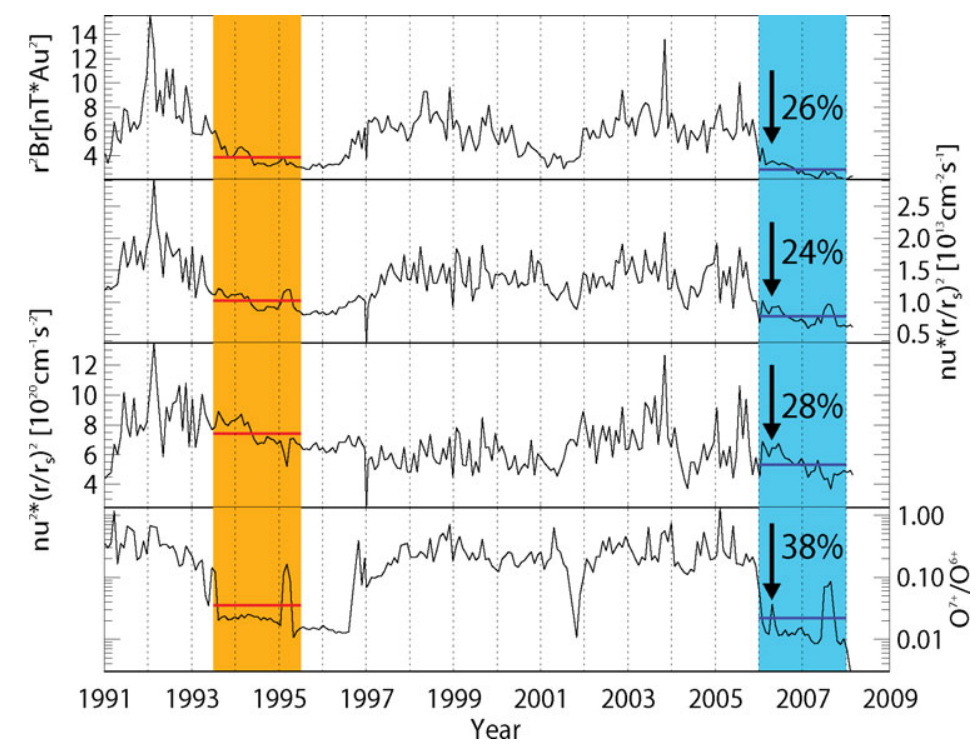

Figure 1. Normalized radial component of the heliospheric magnetic field, solar wind mass flux, solar wind ram pressure, and charge states of O, as observed by Ulysses from 1991 to 2008. 
corona, when the density becomes sufficiently low. Thus, in the current solar minimum, the coronal electron temperature is lower than in the previous minimum.

Gloeckler et al. (2003) found a remarkable correlation between the coronal electron temperature as determined by solar wind charge states and the solar wind speed. For a period in 1996-97, they found a linear relationship between the square of the solar wind speed and the inverse of the coronal electron temperature, as determined by the solar wind charge states. In Figure 2, we repeat this analysis for the same time period as used by Gloeckler et al. (2003), and for a period in 2005-07. We find the exact same linear relationship. It should be noted that the linear relationship is most obvious when there are both fast and slow wind present in the analyzed period. The two time periods chosen are ideal for this. In other time periods, such as the current solar minimum, when the charge states and thus the coronal electron temperature are low, there is a narrower range of solar wind speeds that can be analyzed, and the relationship is less obvious in the data.

We have then several observations to explain: (1) The variation of the normalized radial component of the heliospheric magnetic field, or equivalently the average open magnetic flux of the Sun, during the solar cycle, including the lower value in the current minimum. (2) The correlation of the solar wind mass flux with the normalized radial component of the heliospheric magnetic field. (3) The linear relationship between the square of the solar wind flow speed and the inverse of the coronal electron temperature, and why it is the same during the two different periods shown in Figure 2.

\section{The Behavior of the Heliospheric Magnetic Field during the Solar Cycle}

The Ulysses mission to date has observed the solar magnetic cycle for $\sim 17$ out of the $\sim 22$ years required for a full cycle. The picture that has emerged is one of remarkable simplicity: the magnetic field in the heliosphere appears to be organized into two regions of opposite polarity separated by a single current sheet, which appears to persist throughout the solar cycle (Smith et al. 2001; Jones et al. 2003). In past solar cycles, the average strength of the solar magnetic field that opens into the heliosphere has appeared

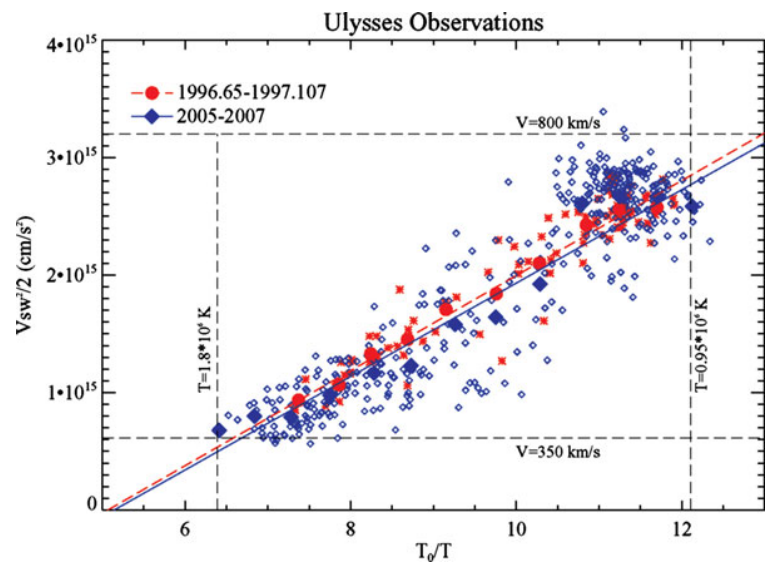

Figure 2. The anticorrelation between the solar wind speed squared and the coronal electron temperature as measured by solar wind charge states (after Gloeckler et al. 2003). Two time periods are shown. The first is the same as in the Gloeckler et al. (2003) analysis; the second is closer to the current solar minimum. 
to be relatively constant, particularly if you compare the field in successive solar minima, and it increases only by a factor of $\sim 2$ at solar maximum (e.g., Wang et al. 2000). The current sheet becomes tilted relative to the solar equator as the solar cycle progresses and rotates over. In this simple picture then it is the rotation of the current sheet that accomplishes the field reversal of the Sun, although the more commonly accepted view is that the field reversal occurs by polar field annihilation as in the Babcock (1961) model (e.g., Wang \& Sheeley 2003).

The organization and the constancy of the heliospheric magnetic field are related. To eliminate heliospheric magnetic flux, it is necessary that magnetic flux of opposite polarity reconnects, forming an inverted ' $U$ '-shaped loop that is convected out of the heliosphere by the solar wind (Fisk \& Schwadron 2001). Such reconnection can only occur at the single current sheet, where magnetic fields of opposite polarity can interact, and within the Alfven radius, which occurs at $\sim 10 r_{S u n}$. Here, a solar loop will also be formed that can return to the Sun, with a net loss of magnetic flux to the heliosphere. While this process is possible, the inverted ' $U$ ' loop should be devoid of heat flux, a so-called heat flux dropout (McComas et al. 1989, 1992). Such dropouts are rarely observed (Lin \& Kahler 1992; Pagel et al. 2005), although some controversy remains in the interpretation of the heat-flux dropout data (Pagel et al. 2007).

The concept that magnetic flux in the heliosphere cannot be readily eliminated has provided a natural explanation for why the heliospheric magnetic field has appeared to return to the same magnitude at successive solar minima, as documented by Svalgaard \& Cliver (2007). There is a background level of magnetic flux always present in the heliosphere. The increases in the heliospheric magnetic field at solar maximum are attributed to an enhanced rate of CMEs. CMEs drag additional magnetic flux into the heliosphere, and if left unabated would result in magnetic flux in the heliosphere that increases without bound (Gosling 1975; McComas 1995). Since this does not occur, it is believed that the magnetic field in the CME reconnects with the background open magnetic flux in a process that has been labeled interchange reconnection (Gosling et al. 1995; Fisk \& Schwadron 2001; Crooker et al. 2002). The CME flux is then converted into open flux, with no net increases in the heliospheric magnetic field. Interchange reconnection can take time to execute, and thus during solar maximum, when the rate of CMEs is high, there is a temporary increase in the heliospheric magnetic field (by a factor $\sim 2$ ); at solar minimum, the rate of CMEs is lower and the heliospheric field returns to its background level (Owens \& Crooker 2006).

As can be seen in Figure 1, unlike our expectations, the normalized radial component of the heliospheric magnetic field, and thus the average open magnetic flux of the Sun, is lower in the current minimum, compared with the previous minimum. The simplest explanation is that we do not know the actual strength of the background, constant level of open flux. In the above argument, we assumed there was no significant contribution of CMEs during solar minimum, and the background level is attained in each minimum. Perhaps that is not the case. If there was still a CME contribution in each previous minimum, but in the current minimum, which is unusually quiet, this contribution is smaller, then we are closer to the background level now than we were previously.

In the remaining sections of this paper, we will consider theories for the solar wind in which open magnetic flux interacts with coronal loops; material is released to provide the mass flux of the solar wind; and the open magnetic flux is displaced resulting in waves and turbulence that heat and accelerate the solar wind. The basis for this theory of the solar wind is the model for the interaction of coronal loops with open flux developed by Fisk (2005). As is illustrated in Figure 3, small loops are emitted through the solar surface. The ends of the loops migrate to the network lanes, where they can reconnect 
with and thus coalesce with other loops. They can also encounter open flux. If the end of the loop has opposite polarity to the open field line, reconnection occurs. The loop is destroyed and the open field line is displaced. There is a small loop formed at the reconnection site, which subducts back into the photosphere.

Consider what happens to loops that emerge under the current sheet. If the emerging loop is oriented such that it is aligned with the polarity of the open flux, then the end points of the loop never encounter open magnetic flux of opposite polarity. The loop grows without bound. One could even argue that this process provides an origin for all large streamer belt loops.

For our purposes here, there is a natural mechanism whereby small loops could grow without bound around the current sheet, and eventually be continuously emitted as large loops into the heliosphere, even during solar minimum. These large loops would also be subject to interchange reconnection with the background level of open magnetic flux, and the addition of magnetic flux in the heliosphere would not have unlimited growth. However, while these loops are present in the heliosphere, before interchange reconnection, they would raise the level of magnetic flux in the heliosphere above the background level, even at solar minimum.

There is still, however, an electron heat flux problem. Loops are identified in the solar wind by counterstreaming, bi-directional electron fluxes (Gosling et al. 1987). There is no particular observational evidence to suggest continuous bi-directional electron fluxes around the current sheet (Zurbuchen \& Richardson 2006). We need to remember, however, that bi-directional electrons, as a measure of loops in the solar wind, are mainly observed in large CMEs. Perhaps the signature is not as clear in the loops we are arguing could be continuously emitted around the current sheet. Certainly the geometry of the expansion is different between current sheet loops and large CMEs. The former have particularly long legs compared to the lateral portion of the loop, which must intercept and be influenced by the current sheet. Whether this will affect the bi-directional electron

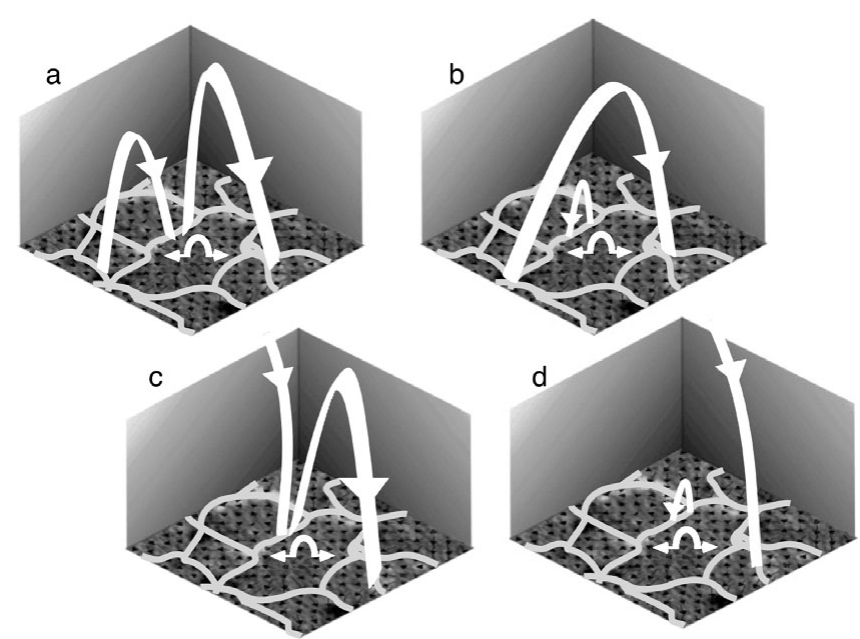

Figure 3. Small loops emerge in the center of supergranules. Each end expands and enters the network lanes, where they move with the random convective motions of the photosphere. In (a) two end points of loops collide and reconnect, coalescing into the large loop shown in (b). In (c), the end point of a loop and an open field line reconnect. In (d), the open file line is displaced to lie over the opposite side of the loop, and the original loop is destroyed. Small loops are created at the reconnection sites, and are assumed to subduct into the photosphere. 
fluxes is a problem that could use more theoretical work, and perhaps a closer look at the observations.

Thus, from the observations in Figure 1, and the above arguments, there is a correlation between the normalized radial component of the heliospheric magnetic field and thus the average open magnetic flux, and the rate of emergence of new magnetic flux on the Sun. The correlation is clear at solar maximum, when the emergence of large active regions result in large CMEs. However, there also appears to be a correlation between the level of open flux present in the absence of large CMEs and the rate emergence of small loops on the Sun, which grow under the current sheet and expand into the heliosphere. We would argue that in the current minimum this rate of emergence of small loops is lower, with a resulting lower level of open magnetic flux in the heliosphere, as seen in Figure 1 , perhaps even a level that approaches the constant, background level of open magnetic flux.

\section{The Behavior of the Solar Wind during the Solar Cycle}

There are two basic types of solar wind acceleration theories. The first is the basic Parker theory, which has been elaborated upon and incorporated into many, quite detailed models for the acceleration of the solar wind (e.g., Parker 1958; Isenberg 1991 and references therein; Marsch 1995 and references therein; Hansteen \& Leer 1995; Axford \& McKenzie 1997; Cranmer et al. 2007). In these models you assume there is a deposition of energy and perhaps momentum into the solar corona. This deposition accelerates the solar wind and determines all other flow parameters, such as the solar wind mass flux. In the second type of solar wind acceleration theory, you assume that the mass flux of the solar wind is determined independently of the acceleration (Fisk et al. 1998, 1999; Fisk 2003). Matter is released from coronal loops as a result of reconnection with open magnetic flux, and this process determines the mass flux. The temperature and density in the corona then adjust to satisfy two independent constraints, the mass flux and the energy deposition, and result in the required supersonic flow.

In the latter theory, the solar wind is created as a result of the reconnection of open magnetic flux with coronal loops. In coronal holes, there is ample open flux present, which reconnects with the cool, small loops present at the base of the coronal hole. There should also be open flux present outside of coronal holes. Fisk \& Zurbuchen (2006) showed that the transport of open magnetic flux resulting from reconnections with coronal loops should result in a uniform, radial component of open flux present in the regions outside of coronal holes. This component of open flux will reconnect with the large, hotter coronal loops on the quiet Sun outside of coronal holes. The mechanism for the origin of the solar wind is the same both inside and outside of coronal holes; the difference is the properties, e.g., the temperature of the coronal loops with which the open flux is reconnecting.

The theory in which the solar wind results from reconnections of open flux with coronal loops has several advantages:

(a) It is easy to imagine with this theory, as we shall show, that the mass flux is related to and correlated with the behavior of the open magnetic flux of the Sun, since we concluded in the previous section that the mass flux results from open flux reconnecting with coronal loops.

(b) This theory, in which the matter that is released to form the solar wind originates in coronal loops, also explains the composition of the solar wind. As shown by Feldman et al. (2005), the composition of coronal loops, the enhancements in elements with low first ionization potential and the electron temperatures and thus charge states, are consistent with those of the solar wind. The composition and coronal electron temperatures 
of the fast solar wind resemble those of the small, cooler loops at the base of the coronal holes. The composition and coronal electron temperatures of the slow solar wind closely resemble those of large coronal loops on the quiet Sun outside of coronal holes.

(c) In this theory it is possible to couple the mass flux of the solar wind with the deposition of energy that accelerates the solar wind. The process of reconnecting open magnetic flux with coronal loops displaces the open magnetic flux in the solar corona. This displacement will produce waves and turbulence in the corona, which when damped deposit energy that heats the solar corona and accelerates the solar wind.

(d) This theory is more likely to result in a predictive model for the solar wind, since certain basic flow parameters of the solar wind, e.g., the mass flux and energy deposition can be directly related to observable properties of the Sun, such as the properties of coronal loops.

We should also note that there is now a verified theory for how small loops that emerge on the Sun evolve and interact, through reconnection, with each other and with open magnetic flux. Fisk (2005) developed a relatively simple model for the evolution of coronal loops, based on the transport model of Schrijver et al. (1997) for magnetic flux concentrations in the random convective motions of the photosphere. As is illustrated in Figure 3, a small loop emerges through the photosphere. The end points of the loop migrate to the network lane, where each end point behaves independently. If the end points of two loops of opposite polarity encounter each other they reconnect and the two loops coalesce into one. If the end point of a loop encounters an open field line with opposite polarity, it reconnects, destroys the original loop, and displaces the open field line. At the reconnection sites, small loops are formed, which are assumed to subduct back into the photosphere. This theory can be used to determine the interaction rates between loops and open field lines, and thus the transport properties of open magnetic flux on the Sun, since the random displacements of open field lines due to reconnections with loops will cause the open magnetic flux to diffuse along the solar surface.

The theory of Fisk (2005) made a major prediction, which has now been confirmed by two independent sets of observations. The theory predicted that magnetic flux of a single polarity that is reconnecting with small coronal loops will tend to accumulate in regions where the rate of emergence of new magnetic flux is a local minimum. Thus, coronal holes, which are concentrations of open magnetic flux, are predicted to occur in regions where the rate of emergence of new magnetic flux is a local minimum. In Abrahmenko et al. (2006) this prediction was confirmed. In a study of 34 coronal holes, the coronal holes were found to occur in regions where the rate of emergence of new magnetic flux is a factor $\sim 2$ lower than the surrounding regions. In Hagenaar et al. (2008) the more general prediction of the theory was verified. Regions of unipolar magnetic flux, whether in coronal holes or from nearby decaying active regions, tend to occur where the rate of emergence of new magnetic flux is a local minimum.

We thus develop our theory to explain the solar wind observations in Figure 1 \& Figure 2 based upon the following concepts: (1) The solar wind mass flux is determined by the release of matter when open magnetic flux reconnects with a coronal loop. (2) The deposition of energy into the corona, which accelerates the solar wind, is the result of the displacement of open magnetic flux due to reconnection of open flux with coronal loops. (3) The evolution and other properties of coronal loops and how they interact are determined by the theory of Fisk (2005).

Solar wind mass flux. In our theory for the solar wind, the mass flux is determined by the amount of material that is released from coronal loops and the frequency with which it is released by reconnection with open magnetic flux. Suppose that the mass that is released from an average loop is $M_{l}$ and the surface number density of loops is $N_{l}$. In 
Fisk (2005), the collision frequency, and thus the reconnection frequency, between a loop and an open magnetic field line containing equal amount of magnetic flux as the loop is (Schrijver et al. 1997)

$$
\frac{1}{\tau_{l, o}}=\frac{3}{4} \frac{\delta h^{2}}{\delta t} N_{o} .
$$

Here, $\delta h^{2} / 2 \delta t$ is the diffusion coefficient due to random convective motions in the photosphere with scale size $\delta h, N_{0}$ is the surface number density of open field lines. It follows then that the time-averaged mass flux of the solar wind is given by

$$
\rho_{s w} u_{s w} S=M_{l} N_{l}\left(\frac{3}{4} \frac{\delta h^{2}}{\delta t}\right) N_{o} S_{s u r f} .
$$

Here, $\rho_{s w}$ is the mass density of the solar wind, $u_{s w}$ is the mean flow speed of the solar wind, and $S$ is the cross section of a solar wind flux tube. Since the time-averaged mass flux of the solar wind should be constant along the flux tube, the left side of equation (4.2) can be evaluated at any location, including at heliocentric distance, $r$. The right side of equation (4.2) is evaluated on the solar surface, where $S_{\text {surf }}$ is the cross section of the flux tube.

The average open magnetic flux within $S_{\text {surf }}$ is $B_{o}=\phi N_{o}$, where $\phi$ is the magnetic flux in an open field line, equal to the magnetic flux in a loop with which the open field line is reconnecting. The total magnetic flux is constant within the flux tube, or $B_{o} S_{\text {surf }}=B_{r} S_{r}$, where $B_{r}$ is the average magnetic field strength normal to the surface $S_{r}$ at heliocentric distance $r$. Recall that the radial component of the heliospheric magnetic field is independent of latitude and longitude. We find that $B_{r} S_{r} \propto B_{r} r^{2}$, the quantity shown in Figure 1, and thus equation (4.2) is consistent with the observed correlation between mass flux and normalized radial magnetic field strength.

In Figure 4, we consider the relationship between the mass flux and the normalized radial magnetic field strength during the same time periods considered in Figure 2, when the Gloeckler et al. (2003) anticorrelation between solar wind speed and coronal electron temperature is particularly evident. One-hour values of the mass flux data are binned into ranges of $B_{r} r^{2}$ and then averaged within the bin into a single point. This process removes the variations in the mass flux that will be introduced by the other parameters in equation (4.2) besides $B_{r} r^{2}$. Clearly, there is a strong linear relationship between mass flux and $B_{r} r^{2}$, as predicted in equation (4.2). Note also that there is no non-zero intercept in the linear relationship. This suggests that the entire mass flux is due to the processes described by equation (4.2), as opposed to, e.g., a portion of the mass flux being due only to the acceleration process, as in a standard solar wind model.

The flow speed and charge states of the solar wind. Consider the deposition of energy into the corona. In our theory for the acceleration of the solar wind the source of energy is the random displacements of open field lines due to reconnections with coronal loops. As discussed in Fisk (2003), such random displacements should introduce magnitude variations in the coronal magnetic field comparable to the average magnetic field strength. When these variations are damped there is deposition of energy into the corona comparable to the energy in the average magnetic field of the corona. The characteristic timescale for the deposition should be the characteristic timescale at which open magnetic field lines reconnect with coronal loops, which using equation (4.1), is

$$
\frac{1}{\tau_{o, l}}=\frac{3}{4} \frac{\delta h^{2}}{\delta t} N_{l} .
$$




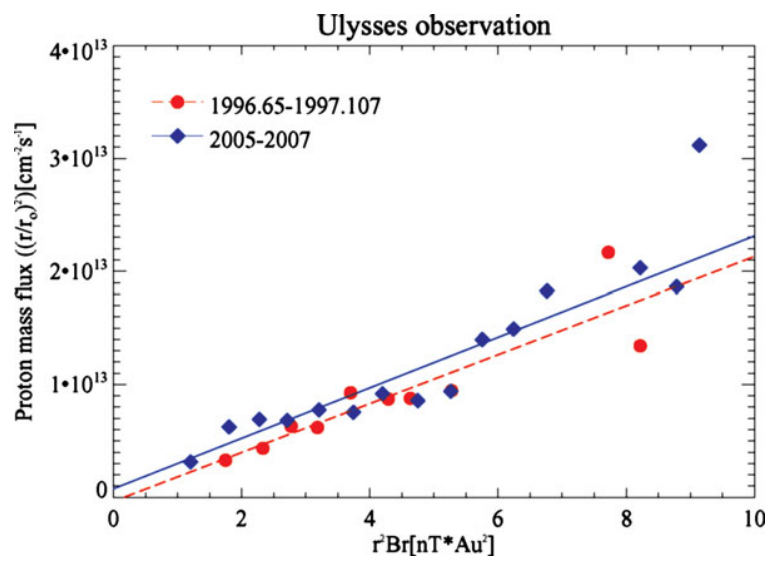

Figure 4. The mass flux of the solar wind versus the bin-averaged, normalized radial component of the heliospheric magnetic field, for the same time periods as shown in Figure 2. The binning technique is described in the text.

In Fisk (2003), the total magnetic energy in a flux tube in the corona is shown to be

$$
W_{\text {mag }}=\frac{1}{8 \pi} \phi N_{o} S_{\text {surf }} \bar{B}_{o} r_{S u n} .
$$

Here $\bar{B}_{o}$ is the average radial component of the open magnetic flux on the solar surface, i.e., $\bar{B}_{o}=B_{r}\left(r^{2} / r_{\text {Sun }}^{2}\right)$, where $r_{S u n}$ is a solar radius; again, $\phi$ is the average magnetic flux in an emerging loop or an open magnetic field line. The only assumption in equation (4.4) is that in the solar corona the magnetic field behaves roughly as a potential magnetic field, with zero current.

The conservation of energy equation of the supersonic solar wind is

$$
\frac{\rho_{s w} u_{s w}^{3} S}{2}=\frac{W_{m a g}}{\tau_{o, l}}-\rho_{s w} u_{s w} S \frac{G M_{o}}{r_{S u n}} .
$$

Here, $G M_{o} / r_{\text {Sun }}$ is the potential energy per unit mass of the Sun. Thus, substituting in equations (4.2), (4.3) and (4.4) into equation (4.5), we derive that

$$
\frac{u_{s w}^{2}}{2}=\frac{\phi}{M_{l}}\left(\frac{\bar{B}_{o} r_{S u n}}{8 \pi}\right)-\frac{G M_{o}}{r_{\text {Sun }}} .
$$

Fisk (2003) noted that the mass contained in loops should be roughly proportional to their temperature, since the density scale height is proportional to the temperature. Thus, $M_{l}=M_{l, \text { ref }}\left(T / T_{\text {ref }}\right)$ where $M_{l, \text { ref }}$ is the mass of a loop at a reference temperature $T_{\text {ref }}$ and $T$ is the temperature of a loop. Further, electron heat flux is large in the corona, and it is readily imaginable that the loops serve as a thermal bath controlling the coronal electron temperature. Thus, the temperature of the loops with which the open flux is reconnecting determines the coronal electron temperature, and the solar wind charge states. Equation (4.6) can then be written as

$$
\frac{u_{s w}^{2}}{2}=\frac{\phi}{M_{l, \text { ref }}}\left(\frac{T_{r e f}}{T}\right)\left(\frac{\bar{B}_{o} r_{S u n}}{8 \pi}\right)-\frac{G M_{o}}{r_{S u n}} .
$$

Equation (4.7) is consistent with the results shown in Figure 2. The intercept on the two linear curves in Figure 2 are $-G M_{o} / r_{S u n}$, and the linear relationship is proportional to $T_{\text {ref }} / T$. The values of $\bar{B}_{o}$ differ slightly in the two time frames shown in Figure 2 , but 
the slopes are essentially the same. This suggests that there is a relationship between $M_{l, \text { ref }}$ and $\bar{B}_{o}$.

\section{Concluding Remarks}

We have examined the behavior of the heliospheric magnetic field and the solar wind during the solar cycle, including the current and previous solar minimum, and used these data to test and refine our previously developed theories:

(a) In previous theories (e.g., Fisk \& Schwardon 2001) we assumed that there is a constant background level of open magnetic flux present in the heliosphere, and that the heliospheric field is reduced to this background level in solar minimum. To explain the reduction in open flux below previous levels in the current minimum, we suggest that there is continuous emission of small loops around the current sheet, even in solar minimum, and that there is less such emission during the current, unusually quiet minimum. While this is a possible explanation it is not the only explanation and additional theoretical and observational consideration is required.

(b) In previous theories (e.g., Fisk \& Schwardon 2001) we assumed that the mass flux of the solar wind is determined by the release of material from loops. There appears to be strong support for this process, at least in the time periods when the Gloeckler et al. (2003) anticorrelation between solar wind speed and coronal electron temperature holds.

(c) In previous theories (e.g., Fisk 2003) we were able to explain the anticorrelation between the solar wind speed and the coronal electron temperature observed by Gloeckler et al. (2003). These concepts, applied to the solar wind acceleration model developed here, appear to be applicable at least in some different periods throughout the solar cycle.

The relationship for the solar wind speed in equation (4.7) holds some promise for being able to predict the solar wind speed based on observed coronal properties. The main dependence is the temperature of the loops from which the material is released to form the solar wind. In principle, loop temperatures are observable. Although it will be important to be able to correctly map the open magnetic flux from the solar surface into the heliosphere, so that the correct loops, those at the base of the open flux, can be determined. A simple potential field source surface model will not do for this mapping since it cannot determine the correct mapping of open flux into primarily closed field regions. Mapping techniques such as the one developed by Gilbert et al. (2008) may be required.

\section{Acknowledgements}

This work was supported in part by the Heliophysics Theory Program, by NASA TR\&T grant NNG056M53G, by NASA/JPL contract 1268016, and by NSF grant ATM 0632471.

\section{References}

Abramenko, V. I., Fisk, L. A., \& Yurhyshyn, V. B. 2006, ApJ(Letters), 641, L65

Axford, W. I. \& McKenzie, J. F. 1997, in: J. R. Jokipii, C. P. Sonnet \& M. S. Giampapa (eds.), Cosmic Winds in the Heliosphere (Tucson: Univ. of Arizona Press), p. 31

Babcock, H. W. 1961, ApJ, 133, 572

Balogh, A. \& Smith, E. J. 2001, Space Sci. Revs., 97, 147

Cranmer, S. R., van Ballegooijen, A. A., \& Edgar, R. J. 2007, ApJS, 171, 520

Crooker, N. U., Gosling, J. T., \& Kahler, S. W. 2002, J. Geophys. Res., 107, 1028

Feldman, U., Landi, E., \& Schwadron, N. A. 2005, J. Geophys. Res., 110(A7), A07109 
Feldman, U., Widing, K. G., \& Warren, H. P. 1999, ApJ, 522, 1133

Fisk, L. A. 1996, J. Geophys. Res. 101(A7), 15,547

Fisk, L. A. 2001, J. Geophys. Res., 106(A8), 15,849

Fisk, L. A. 2003, J. Geophys. Res., 108 (A4), p. SSH 7-1

Fisk, L. A. 2005, ApJ, 626(1), 563

Fisk, L. A. \& Schwadron, N. A. 2001, ApJ, 560(1), 425-438

Fisk, L. A. \& Zurbuchen, T. H. 2006, J. Geophys. Res., 111, A09115

Fisk, L. A., Schwadron, N. A., \& Zurbuchen, T. H. 1998, Space Sci. Revs., 86(1/4), 51-60

Fisk, L. A., Schwadron, N. A., \& Zurbuchen, T. H. 1999c, J. Geophys. Res., 104(A9), 19,765

Fisk, L. A., Zurbuchen, T. H., \& Schwadron, N. A. 1999a, ApJ, 521(2), 868-877

Fisk, L. A., Zurbuchen, T. H., \& Schwadron, N. A. 1999b, Space Sci. Revs., 87(1/2), 43-54

Fisk, L. A., Schwadron, N. A., \& Zurbuchen, T. H. 1999c, J. Geophys. Res., 104(A9), 19,765

Gilbert, J. A., Zurbuchen, T. H., \& Fisk, L. A. 2007, ApJ, 663, 583

Gloeckler, G., Zurbuchen, T. H., Geiss, J. 2003, J. Geophys. Res., 108, SSH 8-1

Gosling, J. T. 1975, Rev. Geophys., 13, 1053

Gosling, J. T., Baker, D. N., Bame, S. J., Feldman, W. C., Zwickl, R. D., \& Smith, E. J. 1987 J. Geophys. Res., 92, 8519

Gosling, J. T., Birn, J., \& Hesse, M. 1995, Geophys. Res. Lett., 22, 869

Hagenaar, H. J., DeRosa, M. L., \& Schrijver, C. J. 2008, ApJ, in press

Hansteen, V. H., \& Leer, E. J. 1995, J. Geophys. Res., 100, 21577

Isenberg, P. A. 1991, in: J. A. Jacobs (ed.), Geomagnetism (San Diego: Academic), vol. 4, p. 1

Jones, G. H., Balogh, A., \& Smith, E. J. 2003, Geophys. Res. Lett., 30, 2

Marsch, E., von Steiger, R., \& Bochsler, P. 1995, A\&GA, 301, 261

Lin, R. P. \& Kahler, S. W. 1992, J. Geophys. Res., 97, 8203

McComas, D. J. 1995, Rev. Geophys., 33, 603

McComas, D. J., Gosling, J. T., \& Phillips, J. L. 1992, J. Geophys. Res., 97, 171

McComas, D. J., Gosling, J. T., Phillips, J. L., Bame, S. J., Luhmann, J. G., \& Smith, E. J. 1989, J. Geophys. Res., 94, 6907

Owens, M. J. \& Crooker, N. U. 2006, J. Geophys. Res., 111, A10104

Pagel, C., Crooker, N. U., Larson, D. E., Kahler, S. W., \& Owens, M. J. 2005, J. Geophys. Res., 110, A01103

Pagel, C., Gary, S. P., de Koning, C. A., Skoug, R. M., \& Steinberg, J. T. 2007, J. Geophys. Res., 112, A04103

Parker, E. N. 1958, ApJ, 128, 664

Schrijver, C. J., Title, A. M., van Ballegooijen, A. A., Hagenaar, H. J., \& Shine, R. A. 1997, ApJ, 487, 424

Smith, E. J. \& Balogh, A. 1995 J. Geophys. Res., 105, 27217

Smith, E. J., Balogh, A., Forsyth, R. J., \& McComas, D. J. 2001, Geophys. Res. Lett., 28, 4159

Svalgaard, L. \& Cliver, E. W. 2007, ApJ(Letters), 661, L203

Wang, Y.-M., Lean, J., \& Sheeley, N. R., Jr. 2000, Geophys. Res. Lett., 27, 505

Wang, Y.-M. \& Sheeley, N. R., Jr. 2003, ApJ, 599, 1404

Zurbuchen, T. H. \& Richardson, I. G., 2006, Space Sci. Revs., 123, 31

Zurbuchen, T. H., Schwadron, N. A., \& Fisk, L. A. 1997, J. Geophys. Res., 102 (A11), 24, 175

\section{discussion}

Usoskin: What kind of behavior would you expect from the background open flux during the Maunder minimum?

FISK: Disconnection of open flux at the simple current sheet that occurs in the heliosphere appears to be difficult. If there was a simple current sheet with limited disconnection for all cycle back to the Maunder minimum, then the same background level of open 
flux currently present in the heliosphere should have been present during the Maunder minimum as well.

BOCHSLER: Are you implying that the solar wind would work the same way if there were no helium?

Fisk: Our theory for the solar wind assumes the mass flux is determined by the process by which material is released from loops by reconnection with open magnetic flux. There is no special role for helium.

VRŠNAK: Does the formula for $V_{S W}^{2} \sim 1 / T$ hold also for coronal holes, i.e., does it cover a proper velocity range (say $300-800 \mathrm{~km} / \mathrm{s}$ )?

Fisk: Yes, the temperature of loops under coronal holes is low, and the resulting solar wind speed is high, in our formula. The slow solar wind originates from hotter loops in regions outside of coronal holes. In some ways it is remarkable that a simple linear relationship describes both fast and slow solar wind which have substantially different origins. 